Annals of Pure and Applied Mathematics

Vol. 17, No. 2, 2018, 151-155

ISSN: 2279-087X (P), 2279-0888(online)

Published on 25 May 2018

www.researchmathsci.org

DOI: http://dx.doi.org/10.22457/apam.v17n2a1

Annals of

Pure and Applied Mathematics

\title{
1-Modular Dual Nearlattices
}

\author{
Shiuly Akhter ${ }^{1}$ and A.S.A. Noor ${ }^{2}$ \\ ${ }^{1}$ Departmentof Mathematics, Rajshahai University, Bangladesh \\ ${ }^{2}$ Department of ETE, Prime University, Dhaka, Bangladesh \\ Email: shiuly_math_ru@yahoo.com, asanoor100@yahoo.com
}

Received 6 April 2018; accepted 22 May 2018

\begin{abstract}
Jayaram [2] introduced the concept of 0-modular semilattice. Recently, Rahman et al. [4] have introduced the concept of 0-modular Nearlattice. In this paper, we discuss 1-modular dual nearlattice. A dual nearlattice $S$ with 1 is said to be 1-modular if for all $a, b, c \in S$ with $c \geq a$ and $a \vee b=1$ imply $a \vee(b \wedge c)=c$ provided $b \wedge c$ exists. Akhter and Noor [8] have discussed 1-distributive join semilattice. In this paper, we include several characterizations of 1-modular dual nearlattices.
\end{abstract}

Keywords: 1-distributive join semilattice, 1-modular dual nearlattice, prime filter, join prime element, dual atom

AMS Mathematics Subject Classification (2010): 06A12, 06A99, 06B10

\section{Introduction}

Varlet [3] introduced the concept of 0-distributive and 0-modular lattices. A lattice $L$ with 0 is called 0-distributive if for all $a, b, c \in L$ with $a \wedge b=0=a \wedge c$ imply $a \wedge(b \vee c)=$ 0 . A lattice $L$ is called 0 -modular if all $a, b, c \in L$ with $c \leq a$ and $a \wedge b=0$ imply $a \wedge(b \vee c)=c$. Of course, every distributive lattice is both 0-distributive and 0-modular. $[1,2,5,6]$ have studied different properties of 0-distributivity and 0-modularity in lattices and semilattices. Akhter et al. [7] discussed some properties of 1-distributive joinsemilattice. A join-semilattice $S$ with 1 is called 1-distributive if for all $a, b, c \in S$ with $a \vee b=1=a \vee c$ imply $a \vee d=1$ for some $d \leq b, c$. In this paper, we discuss 1modular dual nearlattice and give several nice characterizations of 1-modular dual nearlattice. A dual nearlattice $S$ is a join-semilattice together with the property that any two elements possessing a common lower bound, have a infimum. A dual nearlattice $S$ with 1 is called 1-modular if for all $a, b, c \in S$ with $c \geq a$ and $a \vee b=1$ implya $\vee$ $(b \wedge c)=c$ provided $b \wedge c$ exists.

A lattice $L$ with 1 is called 1-distributive if for all $a, b, c \in L$ with $a \vee b=a \vee$ $c=1$ imply $a \vee(b \wedge c)=1$. A lattice $L$ with 1 is called1-modular if for all $a, b, c \in L$ with $c \geq a$ and $a \vee b=1$ imply $a \vee(b \wedge c)=c$. A lattice $L$ ith 0 is called semi complemented if for any $a \in L,(a \neq 1)$ there exists $b \in L,(b \neq 0)$ such that $a \wedge b=0$. Dually a lattice $L$ with 1 is called dual semi complemented if for any $a \in L,(a \neq 0)$ there exists $b \in L,(b \neq 1)$ such that $a \vee b=1$. A lattice $L$ with 0 and 1 is called complemented if for any $a \in L$ there exists $b \in L$ such that $a \wedge b=0$ and $a \vee b=1$. A 


\section{Shiuly Akhter and A.S.A. Noor}

lattice $L$ with 0 is called weakly complemented if for any distinct elements $a, b \in L$, there exists $c \in L$ such that $a \wedge c=0$ but $b \wedge c \neq 0$ (or vice versa).

Let $S$ be a dual nearlattice. A non-empty subset $F$ of $S$ is called filter if (i) $a, b \in F$ implies there exists $d \leq a, b$ such that $d \in F$

(ii) $\quad a \in F, x \in S$ with $x \geq a$ implies $x \in F$.

A filter $F$ is called proper filter of a dual nearlattice $S$ if $F \neq S$. A proper filter $F$ in $S$ is called prime filter if $a \vee b \in F$ implies $a \in F$ or $b \in F$. For $a \in S$, the filter $F=\{x \in S \mid x \geq a\}$ is called the principal filter generated by $a$. It is denoted by $[a$ ). Let $S$ be a dual nerlattice. A subset $I$ of $S$ is called an ideal if (i) $a, b \in I$ implies $a \vee b \in I$ (ii) $a \in S, i \in I$ with $a \leq i$ implies $a \in I$.

An ideal $I$ of a dual nearlattice $S$ is called prime ideal if $I \neq S$ and $S-I$ is prime filter.

An element $a$ of a nearlattice $S$ is called meet prime if $b \wedge c \leq a$ implies $b \leq a$ or $c \leq a$. An element $a$ of a dual nearlattice $S$ is called join prime if $b \vee c \geq a$ implies either $b \geq a$ or $c \geq a$. A non-zero element $a$ of a lattice $L$ with 0 is an atom if for any $b \in L$ with $0 \leq b \leq a$ implies either $0=b$ or $b=a$. An element $a$ of a dual nearlattice $S$ with 1 is called a dual atom if for any $b \in S$ with $a \leq b \leq 1$ implies $a=b$ or $b=1$.

\section{Main results}

To obtain the main results of this paper we need to prove the following first three theorems and one corollary.

Theorem 1. A dual nearlattice $S$ with 1 is 1-modular if and only if for all $a, b, c \in S$ with $c \geq a, a \vee b=1, a \wedge b=c \wedge b$ imply $a=c$ provided $a \wedge b$ exists.

Proof: Suppose $S$ is 1-modular and $a, b, c \in S$ with $c \geq a, a \vee b=1$. Also let $a \wedge b=$ $c \wedge b$. If $a \wedge b$ exists then $c \wedge b$ exists by the lower bound property. Then $a=a \vee$ $(a \wedge b)=a \vee(b \wedge c)=c$. Conversely, let $a, b, c \in S$ with $c \geq a, a \vee b=1$ and $b \wedge c$ exists. Also let $a \wedge b=c \wedge b$ implies $a=c$. Here $c \geq a \vee(b \wedge c)$ and $b \vee[a \vee$ $(b \wedge c)]=b \vee a=1$. Now, $a \vee(b \wedge c) \geq b \wedge c$, so $b \wedge[a \vee(b \wedge c)] \geq(b \wedge c)$. Also $c \geq a \vee(b \wedge c)$ implies $b \wedge[a \vee(b \wedge c)] \leq(b \wedge c)$ and so $b \wedge c=b \wedge[a \vee(b \wedge c)]$, so by the given conditions $c=a \vee(b \wedge c)$ which implies $S$ is 1-modular.

Theorem 2. A dual nearlattice $S$ with 1 is 1-modular if and only if the interval $[x, 1]$ for each $x \in S$ is 1 -modular.

Proof: If $S$ is 1-modular then trivially $[x, 1]$ is 1-modular for each $x \in S$. Conversely, let $[x, 1]$ is 1-modular for each $x \in S$. Let $a, b, c \in S$ with $a \vee b=1, c \geq a$ and $b \wedge c$ exists. Choose $t=b \wedge c$. Then $a \vee(b \wedge c)=a \vee[(t \vee b) \wedge(t \vee c)]=(t \vee a) \vee[(t \vee b) \wedge$ $(t \wedge c)]=t \vee c=c$ as the interval $[t, 1]$ is 1 -modular.

Corollary 3. A dual nearlattice $S$ with 1 is 1 -distributive if and only if the interval $[x, 1]$ for each $x \in S$ is 1-distributive.

Theorem 4. Let $S$ be a dual nearlattice. Then the intersection of any two filters of $S$ is also a filter. 


\section{1-Modular Dual Nearlattices}

Proof: Let $F, G$ be two filters of a dual nearlattice $S$. Let $a \in F \cap G$ and $b \in S$ with $b \geq a$. Then $a \in F$ and $a \in G$. Since both $F$ and $G$ are filters, so $b \in F$ and $b \in G$. Hence $b \in F \cap G$.

Again let $a, b \in F \cap G$. So $a, b \in F$ and $a, b \in G$. Since $F$ and $G$ are both filters, then there exist $f \in F$ and $g \in G$ such that $f, g \leq a, b$. Let $c=f \wedge g$. Then $c \in F \cap G$, where $c \leq a, b$. Hence, $F \cap G$ is a filter.

Theorem 5. For a dual nearlattice $S$ with 1 , if $I(S)$ is 1-modular, then $S$ is 1-modular.

Proof: Suppose $I(S)$ is 1-modular. Let $a, b, c \in S$ with $a \vee b=1, c \geq a$ and $b \wedge c$ exists. Then $(a] \vee((b] \wedge(c])=(c]$ as $I(S)$ is 1-modular. Thus $(a \vee(b \wedge c)]=(c]$ and so $a \vee(b \wedge c)=c$, which implies that $S$ is 1 -modular.

Theorem 6. A dual nearlattice $S$ with 1 is 1-modular if and only if the lattice of filters of the interval $[x, 1]$ for each $x \in S$ is 0 -modular.

Proof: Let $S$ be 1 -modular. Choose any $x \in S$. Then $[x, 1]$ is also 1 -modular. Let $F, G, H$ be filters of the lattice $[x, 1]$ such that $F \supseteq H$ and $F \cap G=[1)$. Then $F \cap(G \vee H) \subseteq H$ is obvious. Let $h \in H$. Now $F \cap G=[1)$ implies $1=f \vee g$ for some $f \in F$ and $g \in G$. Thus $h \vee f \geq f$ and $f \vee g=1$ imply $f \vee[g \wedge(h \vee f)]=h \vee f$ as $S$ is 1-modular. So $h \vee f \in F \cap(G \vee H)$ and hence $h \in F \cap(G \vee H)$. Therefore, $F \cap(G \vee H)=H$ and so the lattice of filters of $[x, 1]$ is 0 -modular.

Conversely, suppose the lattice of filters of $[x, 1]$ is 0 -modular. Let $a, b, c \in$ $[x, 1]$ such that $c \geq a, a \vee b=1$. Then $[a) \supseteq[c]$ and $[a) \wedge[b]=[1)$. So by 0-modular property, $[a) \wedge([b) \vee[c))=[c)$. Thus, $[a \vee(b \wedge c))=[c)$ and so $a \vee(b \wedge c)=c$. This implies $[x, 1]$ is 1 -modular. Therefore, by theorem $2, S$ is 1 -modular.

Theorem 7. If a dual nearlattice $S$ with 1 is 1 -distributive and $[x, 1]$ is dual semi complemented for each $x \in S$, then the interval $[x, 1]$ is0-distributive for each $x \in S$.

Proof: Let $a, b, c \in[x, 1]$ with $a \wedge b=x=a \wedge c$. Suppose $a \wedge(b \vee c) \neq x$. Then there exists $p \neq 1$ in $[x, 1]$ such that $p \vee(a \wedge(b \vee c))=1$. Then $a \vee p=1=(b \vee c) \vee p$. Thus $p \vee b \vee a=1=(p \vee b) \vee c$. This implies $(p \vee b) \vee(a \wedge c)=1$ as $S$ is 1 distributive. This implies $1=p \vee b \vee x=p \vee b$. Again, using the 1-distributivity of $\mathrm{S}$, $p \vee(a \wedge b)=1$. That is, $1=p \vee x=p$ which gives a contradiction. Therefore, $a \wedge$ $(b \vee c)=x$. Hence, $[x, 1]$ is 0 -distributive.

Theorem 8. Let $S$ be a 1-modular dual nearlattice and $I, J$ are two ideals such that $I \vee J=(1]$ and $I \cap J=(x]$ for some $x \in S$. Then both $I$ and $J$ are principal ideals.

Proof: Suppose $I \vee J=(1]$ and $I \cap J=(x]$ for some $x \in S$. Then $1=i \vee j$ for some $i \in I$ and $j \in J$. Let $b=x \vee i$ and $c=x \vee j$. Then $b \in I$ and $c \in J$. We claim that $I=(b]$

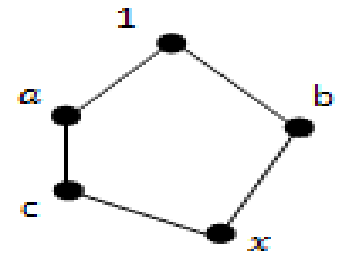

Figure 1: Pentagonal sublattice 
Shiuly Akhter and A.S.A. Noor

and $J=(c]$. Indeed, if for instance, $J \neq(c]$, then there exists $a \in J$ such that $a>c$. Then $\{x, c, a, b, 1\}$ is a pentagonal sublattice of $S$. This implies $S$ is not 1-modular and which gives a contradiction.

Therefore, $J=(c]$. Similarly, $I=(b]$. Hence both $I$ and $J$ are principal ideals.

A dual nearlattice $S$ with 1 is called a dual semi Boolean lattice if it is distributive and the interval $[x, 1]$ for each $x \in S$ is complemented.

Theorem 9. If a section complemented 1-modular dual nearlattice $S$ is1-distributive, then it is dual semi Boolean.

Proof: Let $b<a$ for some $a, b \in S$. Then $b<a \leq 1$. Since $[b, 1]$ is complemented so there exists $c \in[b, 1]$ such that $c \wedge a=b$ and $c \vee a=1$. Now, if $b \vee c=1$, then by the 1-modularity of $S, b=b \vee(c \wedge a)=a$, which is a contradiction. Therefore, $b \vee c \neq 1$. This implies $S$ is weakly complemented. Also since $S$ is 1-distributive, so by Corollary 3, $[x, 1]$ is Boolean for each $x \in S$ and so $S$ is dual semi Boolean.

Lemma 10. In a bounded dual semi complemented lattice $L$, every join prime element is an atom.

Proof: Suppose $a$ is a join prime element. Let $0<b \leq a$. Then $0<b \leq 1$. Since $L$ is dual semi complemented, there exists $c \in L(c \neq 1)$ such that $b \vee c=1$. Since $b \leq a$, so $c \vee a=1$. Since $a$ is join prime element so this implies $c \geq a$ or $b \geq a$. But $c \geq a$ implies $c=c \vee a=1$, which is a contradiction. Hence $b \geq a$ and so $a=b$. Therefore, $a$ is an atom.

Lemma 11. Let $L$ be a bounded 1-modular lattice. If $b \in L$ is an atom and $a \vee b=1$ for some $a \neq 0(a \in L)$, then $a$ is a dual atom.

Proof: Suppose $a \leq c<1$ for some $c \in L$. Since $c \geq a$ and $a \vee b=1$, so by 1modularity, $a \vee(b \wedge c)=c$. Also since $c<1$, it follows that $b>b \wedge c$ and so $b \wedge c=0$ as $b$ is an atom. Consequently, $a=a \vee 0=a \vee(b \wedge c)=c$ by 1-modularity. Thus $a$ is a dual atom.

Lemma 12. Let $S$ be a 1-modular dual nearlattice and $[x, 1]$ is dual semi complemented for each $x \in S$. If for each $x \in S, 1$ is the join of a finite number of join prime elements in $[x, 1]$. Then $x$ is the meet of finite number of dual atoms in $[x, 1]$.

Proof: Let $1=\mathrm{V}_{i=1}^{n} p_{i}$, where $p_{i}{ }^{\prime} s$ are join prime elements in $[x, 1]$. By Lemma 10, each $p_{i}$ is an atom in $[x, 1]$. Since each $p_{i} \neq 1$ and $[x, 1]$ is dual semi complemented, so there exists $q_{i} \in[x, 1]$ such that $p_{i} \vee q_{i}=1, i=1,2, \cdots, n$. Also by Lemma 11, each $q_{i}$ is a dual atom in $[x, 1]$. Let $c=\wedge_{i=1}^{n} q_{i}$. Then $c \wedge p_{i}=x$ as $p_{i}$ is an atom for each $i$. As $[x, 1]$ is dual semi complemented and 1 is the join of finite number of join primes, hence $[x, 1]$ is 1-distributive and so by Theorem 5, $[x, 1]$ is 0-distributive.Therefore, $c \wedge\left(\vee_{i=1}^{n} p_{i}\right)=$ $x$. That is, $c=c \wedge 1=x$. Thus $x=\wedge_{i=1}^{n} q_{i}$.

We conclude this paper with the following Theorem which trivially follows from [2].

Theorem 13. For a dual nearlattice $S$ with $1, S$ is dual semi Boolean if and only if the following conditions are satisfied.

(i) $\quad[x, 1]$ is dual semi complemented for each $x \in S$

(ii) $\quad S$ is 1-modular 


\section{1-Modular Dual Nearlattices}

(iii) 1 is the join of a finite number of join primes

\section{Conclusion}

In this paper, we study the concept of 1-modular dual nearlattice. We also include several characterizations of 1-modular dual nearlattices and prove some results on 1-modular dual nearlattices. Here we prove that, a dual nearlattice $S$ with 1 , is 1 -modular if and only if the lattice of filters of the interval $[x, 1]$ for each $x \in S$ is 0 -modular.

Acknowledgements. The authors are grateful to the reviewers for their valuable comments and suggestions for the improvement of the paper.

\section{REFERENCES}

1. A.S.A.Noor and M.Begum, Some properties of 0 -distributive meet semilattice, Annals of Pure and Applied Mathematics, 2(1) (2012) 60-66.

2. C.Jayaram, 0-modular semilattices, Studia Sci. Math. Hung., 22 (1987) 189-195.

3. J.C.Varlet, A generalization of the notion of pseudo-complementedness, Bull. Soc. Sci. Liege, 37 (1968) 149-158.

4. M.Z.Rahman, A.S.A.Noor and M.B.Rahman, 0-modular nearlattice, Annals of Pure and Applied Mathematics, 3(2) (2013) 100-107.

5. M.Z.Rahman, M.B.Rahman and A.S.A.Noor, 0-distributive nearlattice, Annals of Pure and Applied Mathematics, 2(2) (2012) 177-184.

6. P.Balasubramani and P.V.Venkatanarasimhan, Characterizations of the 0Distributive Lattice, Indian J. Pure Appl. Math., 32(3) (2001) 315-324.

7. S.Akhter, A.S.A.Noor and M.A.Ali, Some properties of 1-distributive join semilattices, J. Mech. Cont. \& Math. Sci., 9(2) (2015) 1377-1384.

8. S.Akhter and A.S.A.Noor, 1-distributive join semilattice, J. Mech. Cont. \& Math. Sci., 7(2)(2013), 1067-1076. 